\title{
A Difference Method of Extending Edge Based on the Mean Value Theorem and the Minimax Principle and Its Application
}

\author{
Zhongxiang Xiao ${ }^{1, a}$, Chunguan Zhang ${ }^{2, b^{*}}$ and Jingguo Chen ${ }^{3, c}$ \\ ${ }^{1}$ College of Electronic Engineering, Xi'an Shiyou University, Xi'an 710065, China \\ ${ }^{2}$ School of Earth Sciences and Engineering, Xi'an Shiyou University, Xi'an 710065, China \\ ${ }^{3}$ Huabei Department, BGP Inc. of CNPC, Renqiu 062552, Hebei, China \\ axzx@xsyu.edu.cn, ${ }^{\mathrm{b}}$ chunguan-zhang@163.com, ${ }^{\mathrm{c}} 1033107340 @ q q . c o m$ \\ *The corresponding author
}

Keywords: Difference method of extending edge; Boundary effect; Panel data; Mean value theorem; Minimax principle.

\begin{abstract}
This research aims to analyze the effect of a difference method of extending edge based on the mean value theorem and the minimax principle and its applications. In this research, the theoretical model of the potential field was designed, and then the theoretical data was calculated. Besides, the data was processed by the difference method of extending edge and the ordinary Kriging method, and the differences were analyzed between the theoretical data and the results of extending edge. Moreover, the effect of this difference method of extending edge was discussed. Results show that the changes of the extending data by the difference method of extending edge is similar to that of the theoretical data, especially when the potential field value of the theoretical data block in the boundary is in a declining trend. The boundary effect is not obvious, indicating that the outward extending data based on the difference method of extending edge are quite reasonable, so that the extending data is suitable for the further processing and inversion.
\end{abstract}

\section{Introduction}

As is known to all, extending edge of potential field data can improve its conversion processing accuracy both in the spatial domain or frequency domain. For the interpolation of irregular data, especially the interpolation problem, the predecessors has done a lot of work $[1,2,3,4]$ and has made a better effect. For the extending edge problem of the potential field data, the predecessors has also studied, and has developed several methods of the extending edge. Duan and his collaborators [5] developed the extending edge method of regional field, and processed the theoretical data and the actual data by the extending edge method of regional field and the discount extending edge method and the extending edge method of cosine decay to zero, and then analyzed these results processed by these three methods. Aimed at the obvious boundary effect of the extending edge method of cosine decay to zero and the discount extending edge method, Ma and his collaborators [6] developed the three-direction extending edge method, and the boundary effect can be reduced to some extent. Although the predecessors has studied the extending edge method of potential field data, but the extending edge effects are poor, so it is necessary to develop a new extending edge method of potential field with more reasonable and practical.

On the basis of detailed analysis of difference interpolation methods and extending edge methods and the mean value theorem and the maximum value and minimum value theorem, the authors develop a new extending edge method of potential field data, design theoretical model, calculate theoretical data, process the actual data by this extending edge method, analyze the results of extending edge, discuss the applied effects of difference extending edge method. 


\section{Method}

The potential field satisfies the conditions of the mean value theorem and the maximum value and minimum value theorem in the passive space, namely the analytic space. Based on these two theorems, the theory of mathematical physics equation gives finite difference solution solving a problem of boundary value problem of elliptic partial differential equation. On the basis of this difference method, the authors develop the difference extending edge method, and the concrete practices are as follows:

Assumption that the potential field value of all boundary points of gridded data are zeros, or equal to the average value of potential field of original data block, while the potential field value of all grid mesh points of the original gridded data remain unchangeable.

As an example to the extending edge of gravity anomaly value, the anomaly values of all the $(i, j)$ points can be calculated by the following formula in outward extending area,

$$
\Delta g(i, j)=\frac{\Delta g(i-1, j)+\Delta g(i+1, j)+\Delta g(i, j-1)+\Delta g(i, j+1)}{4}
$$

Where $i$ is the number of columns of gridded data, and $j$ is the line number of gridded data.

All the outward extending points are calculated once, called once iteration, such in this way, with hundreds of iterations, until all calculated values, $\triangle \mathrm{g}(\mathrm{i}, \mathrm{j})$, are unchangeable. This iterative process is very quick, and certainly converges.

\section{Model Data Analysis}

In order to test the effect of the difference method of extending edge, the authors designed the theoretical model, two spheres with difference density, and then calculated the theoretical gravity anomaly values by the theoretical formula of gravity anomaly. The center coordinates of these two spheres are $(-50 \mathrm{~m},-50 \mathrm{~m},-80 \mathrm{~m})$ and $(50 \mathrm{~m}, 50 \mathrm{~m},-30 \mathrm{~m})$, and the semidiameters are $50 \mathrm{~m}$ and $20 \mathrm{~m}$, and the residual densities of these two spheres are $0.8^{*} 10^{3} \mathrm{~g} / \mathrm{cm}^{3}$ and $-0.8^{*} 10^{3} \mathrm{~g} / \mathrm{cm}^{3}$. According to the above parameters, the authors calculated the theoretical gravity anomaly values of the theoretical model, and the result was shown in Fig. 1. As can be seen from Fig. 1, the anomalies show the maximum value and minimum value above these two spheres, and the absolute values of the anomaly values display an outward reducing trend.

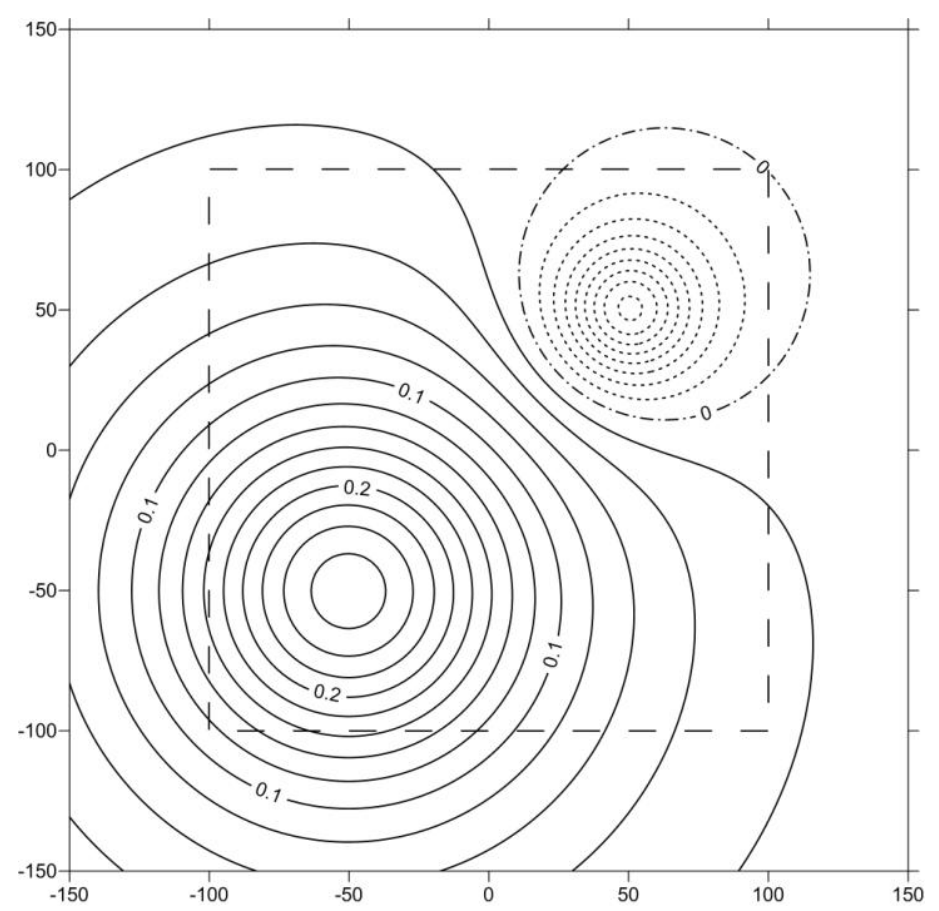

Figure 1. The theoretical gravity anomaly map. The square frame of dotted line is the extent extracting the calculated data, and the value unit is $10^{-5} \mathrm{~m} / \mathrm{s}^{2}$. 
To test the effect of the difference method of extending edge, the authors extracted the data in the square frame of dotted line, and then extended the outward data by the difference method of extending edge (Fig. 2). As can be seen from Fig. 2, the contours are exactly the same between the Fig. 1 and the Fig. 2 in the square frame of dotted line, and the shapes are basically the same between these two figures outside the square frame of dotted line. Although the features of the two figures are similar, but obviously there are differences, and the main difference is the gradient. Obviously, the gradient of the extending data is greater in Fig. 2.

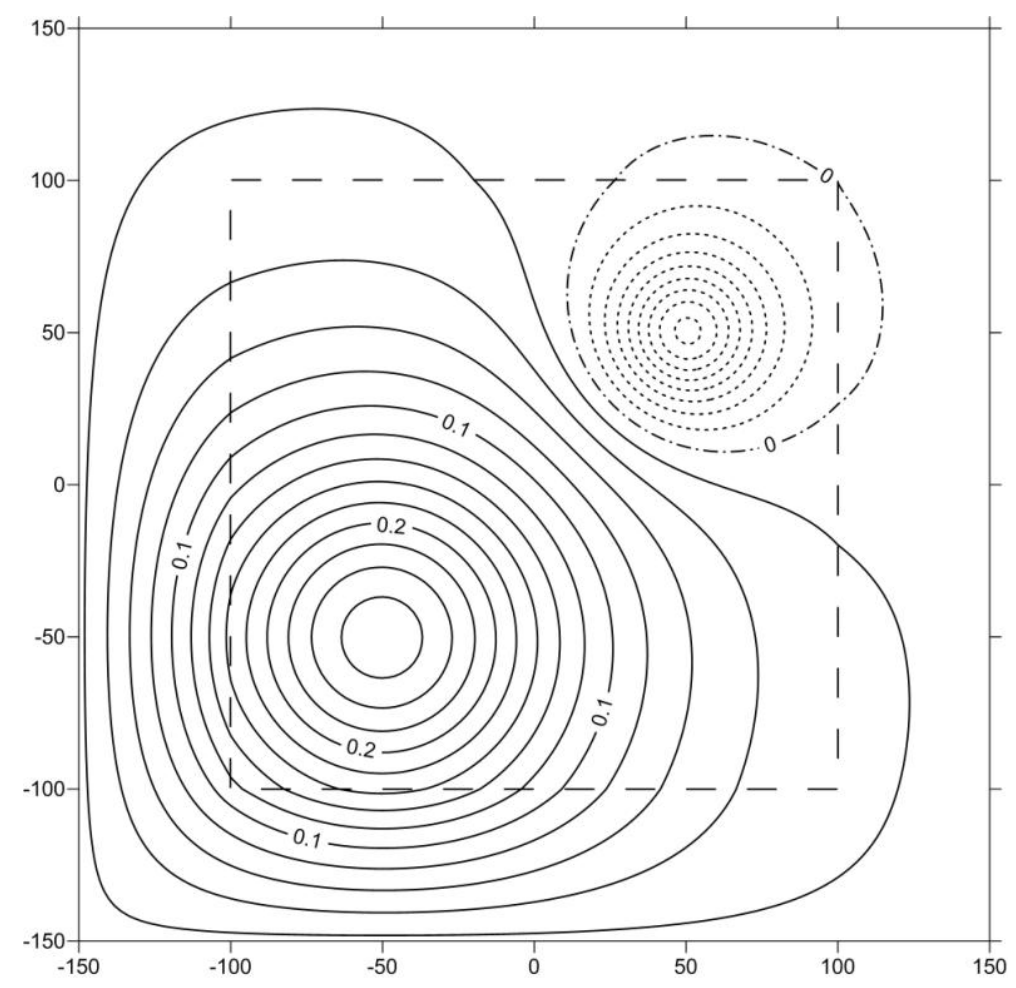

Figure 2. The calculated gravity anomaly map by the difference extending edge method. The square frame of dotted line is the extent extracting the calculated data, and the value unit is $10^{-5} \mathrm{~m} / \mathrm{s}^{2}$.

As a best unbiased interpolation method with minimum estimate variance, the predecessors have done a lot of studies for the ordinary Kriging method [7, 8, 9, 10]. The authors also interpolated by the ordinary Kriging method for the area outside the square frame of dotted line (Fig. 3). As can be seen from Fig. 3, except for the contours being exactly the same between the Fig. 1 and the Fig. 3 in the square frame of dotted line, the shapes show a radiant shape in the Fig. 3 outside the square frame of dotted line, and the features of the extending data display a big difference between the two figures.

The above analysis shows that the outward extending data based on the difference method of extending edge are quite reasonable, so that the extending data is suitable for the further processing and inversion. Meanwhile the boundary effect of the outward extending data based on the ordinary Kriging method is obvious, so that this extending data are not suitable for the further processing and inversion.

\section{Case Analysis}

There are satellite altimetry gravity anomaly data in the sea area of the southwestern Cambodia, but the land area is the blank area of gravity data surrounding the sea area, mainly including the A blank area, the B blank area, and the $\mathrm{C}$ blank area (Fig. 4). In order to get better processing results, it is necessary to fill these blank areas with reasonable gravity data by suitable extending edge method. In this paper, the authors filled these blank areas with the difference method of extending edge based on the mean value theorem and the minimax principle, and the result was shown in Fig. 5. 


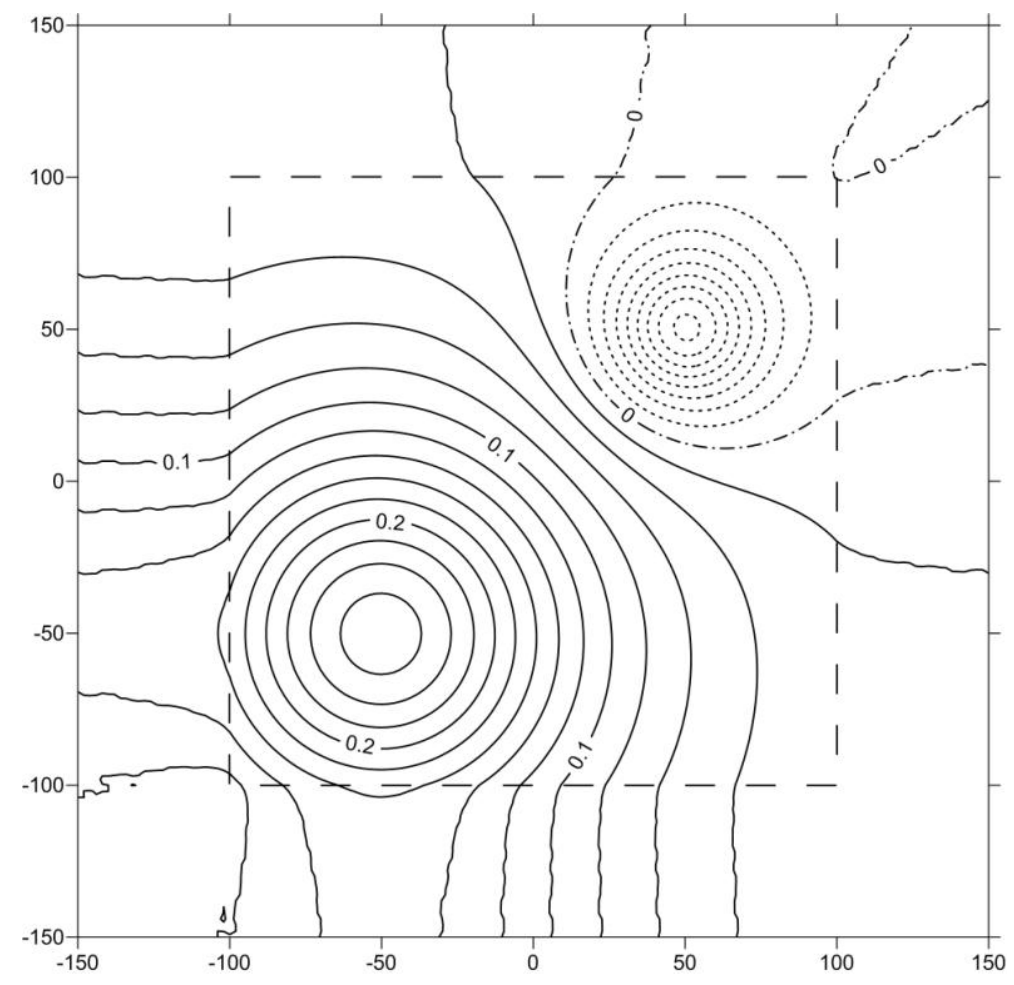

Figure 3. The calculated gravity anomaly map by the ordinary Kriging method. The square frame of dotted line is the extent extracting the calculated data, and the value unit is $10^{-5} \mathrm{~m} / \mathrm{s}^{2}$.

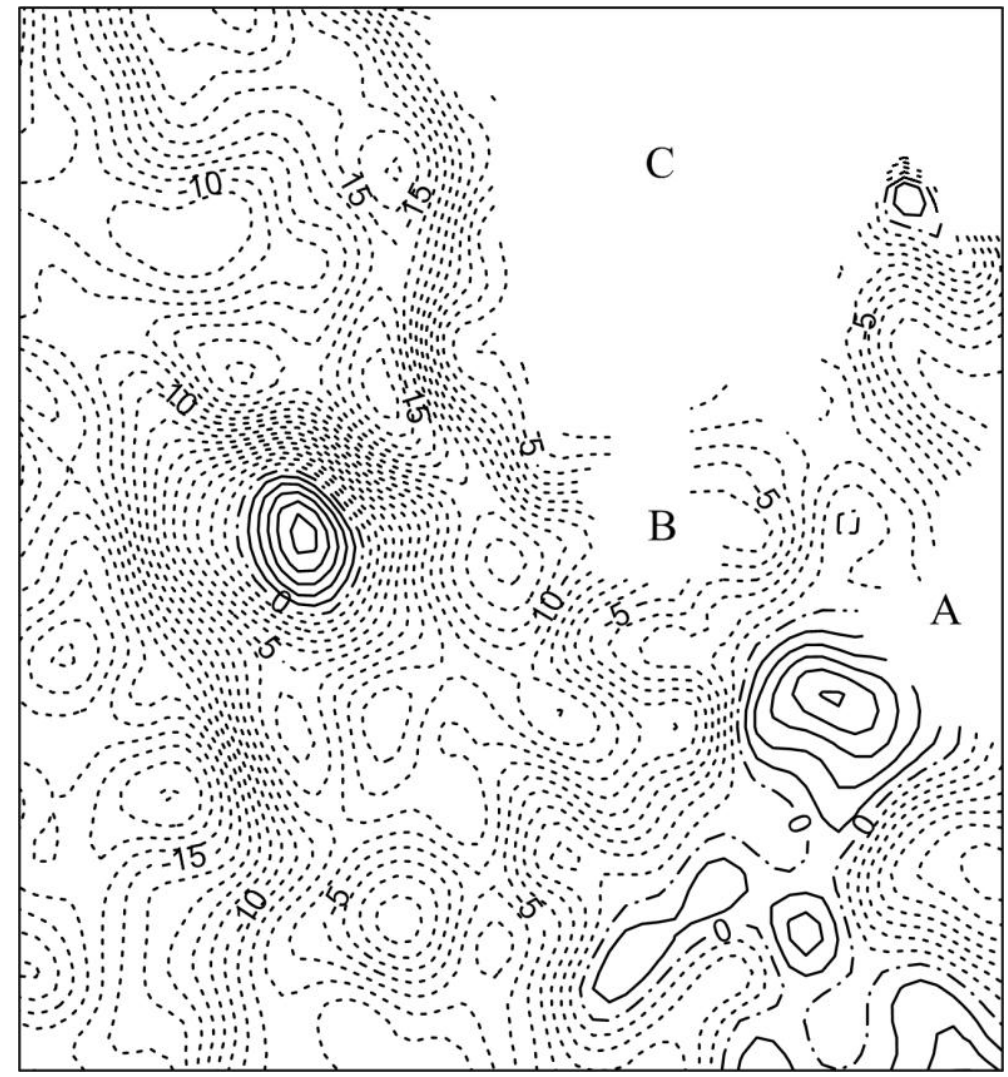

Figure 4. The gravity anomaly map of southwestern Cambodia with blank areas. The value unit is $10^{-5} \mathrm{~m} / \mathrm{s}^{2}$, and A, B, C are the blank areas of gravity data.

As can be seen from Fig. 4, the A area and the B area are more smaller, and there are more satellite altimetry gravity data surrounding these two areas, meanwhile the $\mathrm{C}$ area is larger, and there is less satellite altimetry gravity data surrounding the area. The calculated results show that there are more 
local gravity anomalies in the A area and the B area, and the features of the gravity anomaly display the macro changes in the $\mathrm{C}$ area.

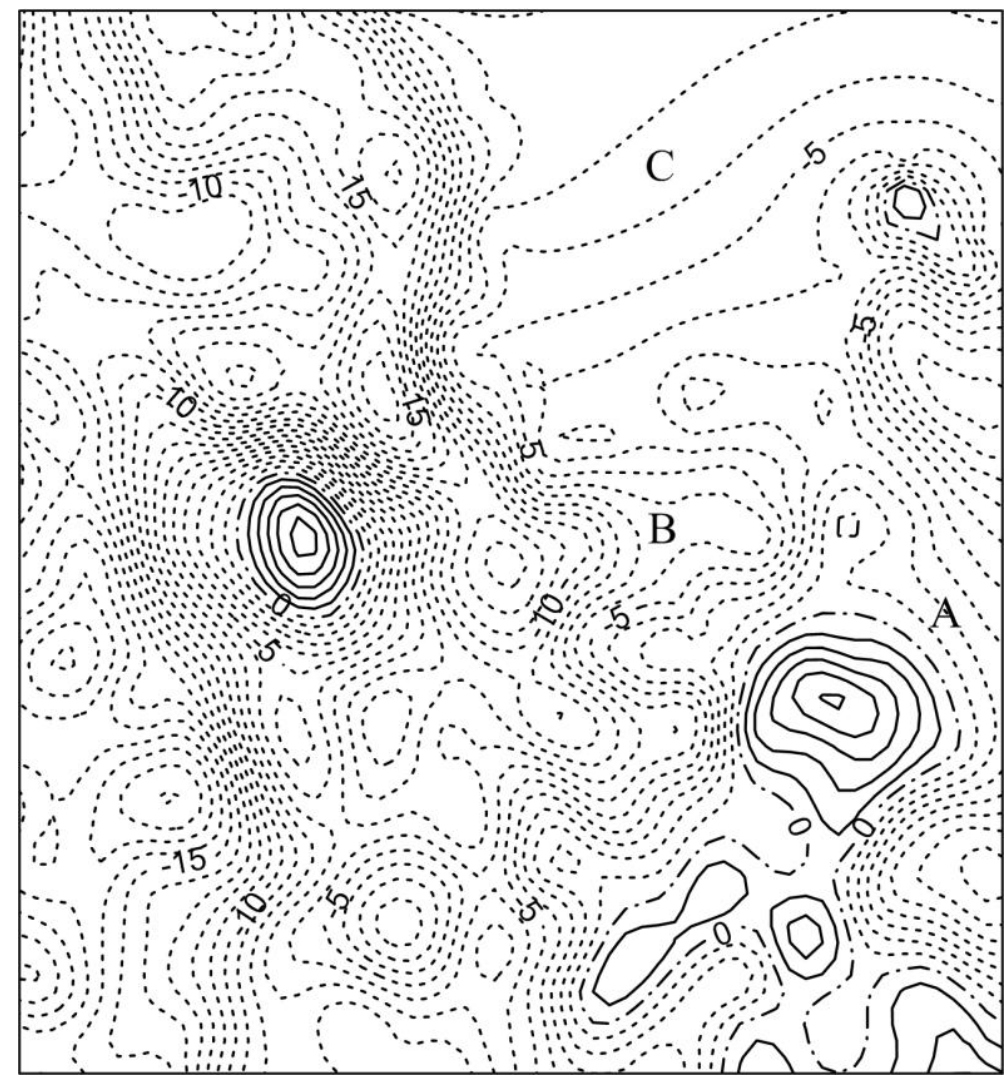

Figure 5. The gravity anomaly map of southwestern Cambodia by the difference extending edge method. The value unit is $10-5 \mathrm{~m} / \mathrm{s} 2$, and there are the calculated data in A, B, C area.

\section{Conclusions}

The changes of the extending data by the difference method of extending edge is similar to that of the theoretical data, especially when the potential field value of the theoretical data block in the boundary is in a declining trend.

The boundary effect is not obvious, indicating that the outward extending data based on the difference method of extending edge are quite reasonable, so that the extending data is suitable for the further processing and inversion.

\section{Acknowledgements}

This work is supported by the Scientific Research Program Funded by Shaanxi Provincial Education Department (No. 14JK1579), China. The authors thank Prof. An Yulin from China University of Geosciences and Prof. Zhang Minghua from China Geological Survey for their guidance and help. We are also grateful for the valuable comments of referees for improving the original manuscript.

\section{References}

[1] L.H. Guo, X.H. Meng, Z.H. Guo, G.F. Liu, J.Y. Chang and G.X. Yu, Gridding methods of geophysical irregular data in space domain, Geophysical \& Geochemical Exploration. 29 (2005) 438-442. 
[2] M.H. Zhang, J.H. Qiao, J.M. Huang, C.X. Wang, G.M. Han, Q. Tian, L. Liu, L. Hu, The soft of gravity, magnetic and electrical data processing and interpretation (RGIS), Geological publishing house, Beijing, 2011

[3] Z.H. Li, Z.C. Luo, H.H. Wang and B. Zhong, Requirements for gravity data within the given accuracy of the interpolated gravity anomaly, Geomatics and Information Science of Wuhan University. 36 (2011) 1328-1331.

[4] W.G. Wu, The application of gridding and blank processing of Surfer: A case study of the mapping data extension processing of 1:50 000 geological stream sediment survey, Geophysical \& Geochemical Exploration. 39 (2015) 602-605.

[5] B.C. Duan and S.Z. Xu, A study of the scheme of extending edge in the processing of separating local field from regional field for magnetic/gravity anomaly, Computing Techniques for Geophysical and Geochemical Exploration. 19 (1997) 298-304.

[6] G.Q. Ma, L.S. Meng, X.J. Du and F.X. Zhang, The study of edge expansion and optimization median filtering method in magnetic data processing, Computing Techniques for Geophysical and Geochemical Exploration. 32 (2010) 194-199.

[7] G.F. Liu, X.H. Meng, Y.B. Yue, J.Y. Chang and L.H. Guo, The application of keiging to GPS data interpolation, Geophysical \& Geochemical Exploration. 30 (2006) 175-178.

[8] X.P. Gong and Y.H. Yang, Ordinary Kriging algorithm applied for interpolation, Journal of Northwest University (Natural Science Edition). 38 (2008) 878-882.

[9] C. Fan and D.G. Wang, Shape functions in meshless Kriging interpolation method, Journal of Liaoning Technical University (Natural Science). 28 (2009) 589-562.

[10] J. Li, S.H. Li, P. Mao and Y.K. Chen, Selection of number of conditional data in Kriging interpolation, Fault-Block oil \& Gas Field. 17 (2010) 277-279. 